\title{
1 Gene network module changes associated with the vertebrate fin to
}

\section{2 limb transition}

3 Pasan C Fernando ${ }^{1^{*}}$, Paula M Mabee ${ }^{2}$ and Erliang Zeng ${ }^{3,4,5,6^{*}}$

$4{ }^{1}$ Department of Biology, University of South Dakota, Vermillion, SD, USA; Current

5 affiliation: Department of Plant Sciences, University of Colombo, Sri Lanka

62 Department of Biology, University of South Dakota, Vermillion, SD, USA; Current

7 affiliation: National Ecological Observatory Network, 1625 38th St. \#100, Boulder, CO

8 80301, USA

$9{ }^{3}$ Division of Biostatistics and Computational Biology, College of Dentistry, University of

$11{ }^{4}$ Department of Preventive \& Community Dentistry, College of Dentistry, University of

12 Iowa, Iowa City, IA, USA

135 Department of Biostatistics, College of Public Health, University of Iowa, Iowa City, IA,

14 USA

$15{ }^{6}$ Department of Biomedical Engineering, College of Engineering, University of Iowa, Iowa

16 City, IA, USA

17 * Correspondence: pasanfernando@pts.cmb.ac.lk and erliang-zeng@uiowa.edu

19 Keywords: gene networks, gene modules, evolution, fin to limb transition, network

20 comparison 


\section{Abstract}

22 Evolutionary phenotypic transitions, such as the fin to limb transition in vertebrate

23 evolution, result from changes in associated genes and their interactions, often in response to

24 changing environment. Identifying the associated changes in gene networks is vital to achieve a

25 better understanding of these transitions. Previous experimental studies have been typically

26 limited to manipulating a small number of genes. To expand the number of analyzed genes and

27 hence, biological knowledge, we computationally isolated and compared the gene modules for

28 paired fins (pectoral fin, pelvic fin) of fishes (zebrafish) to those of the paired limbs (forelimb,

29 hindlimb) of mammals (mouse) using quality-enhanced gene networks from zebrafish and

30 mouse. We ranked module genes according to their weighted-degrees and identified the highest-

31 ranking hub genes, which were important for the module stability. Further, we identified genes

32 conserved during the fin to limb transition and investigated the fates of zebrafish-specific and

33 mouse-specific module genes in relation to their involvements in newly emerged or lost

34 anatomical structures during the aquatic to terrestrial vertebrate transition. This paper presents

35 the results of our investigations and demonstrates a general network-based computational

36 workflow to study evolutionary phenotypic transitions involving diverse model organisms and

37 anatomical entities. 


\section{Introduction}

Phenotypes, such as fin development and limb development, are the result of multiple genes working together in complex biological pathways [1, 2]. Evolutionary modifications in

47 phenotypes due to environmental or other changes involve rewiring gene interactions and their

48 involvements in pathways [2,3]. Most often, it is likely the network of multiple protein

49 interactions rather than the contribution of a single protein that determines the resulting

50 phenotype $[1,4]$. Therefore, investigating the collection of genes and their interactions, i.e.,

51 modular gene structure [1], underlying phenotypes is important in evolutionary biology to

52 understand the evolutionary mechanisms that drive phenotypic changes. Gene module analysis

53 has become common in bioinformatics, and the concept of modular evolution has emerged to

54 explain the changes in groups of genes rather than a single gene when studying the evolution of

55 organisms [5-7]. However, most of these studies have focused on smaller protein complexes

56 (typically containing less than 20 proteins) that determine molecular functions and biological

57 pathways [8-10]. Phenotypes, such as fin and limb development, are resulted by a large number

58 of proteins having diverse molecular functions and belonging to several biological pathways.

59 Even the few protein network studies that focus on phenotypes have targeted human diseases

$60[11,12]$, and to our knowledge, there have been no evolutionary studies of modules to

61 understand evolutionary phenotypic transitions. As there have been important anatomical

62 changes associated with the vertebrate evolution, such as the fin to limb transition, module

63 evolution studies for anatomical changes are essential to unravel new evolutionary information,

64 which serves as the motivation for our work.

65 The fin to limb transition is an iconic anatomical change associated with the evolution of

66 terrestrial vertebrates from aquatic fish-like ancestors [13, 14]. According to fossil record, the 
67 transformation of fishes into land vertebrates began in the Devonian, 365-408 million years ago

$68[13,15]$. This transformation is associated with many phenotypic changes in addition to the fin to

69 limb transition, including changes in the cranial and axial skeleton [13]. The relationship

70 between homologous anatomical structures of land and aquatic vertebrates is evident from

71 several similar characteristics. For instance, the pectoral fin endoskeleton of panderichthyid fish

72 fossils shows significant similarities with the limb skeletons of terrestrial vertebrates (tetrapods)

73 such as the presence of a proximal humerus and two distal bones [14]. Such evidence indicates

74 that forelimbs and hindlimbs of tetrapods are homologous to pectoral and pelvic fins of fishes,

75 respectively.

76 Identifying the genetic changes associated with the fin to limb transition is a prominent topic

77 in evolutionary biology $[16,17]$. Many wet lab experiments have demonstrated the evolutionary

78 importance of genes such as $\operatorname{sh}[14,16]$. Few computational studies, however, have been

79 targeted on the fin to limb transition [17]. The recent availability of large PPI networks and the

80 ability to perform module analysis through the advancement of network algorithms provide an

81 opportunity for a new perspective on genetic changes associated with the fin to limb transition.

82 Graph theoretic methods are critical to the study of networks in biology. These methods

83 are enabled by biological knowledge that is represented as computational graphs, such as

84 protein-protein interaction (PPI) networks and biological ontologies. In graph theory, a module is

85 defined as a set of nodes that are highly connected internally and sparsely connected with

86 external nodes [1]. These network modules usually correspond to biological functions that

87 contribute to phenotypes; hence, they are often referred to as 'functional modules' in biological

88 vocabulary $[6,18]$. 
There are a number of functional module detection algorithms that can be used to detect

90 modules in a graph [5]. Some methods, such as graph partitioning [19], only consider the

91 network structure and do not require any prior information. For modules that are known to

92 involve a large number of genes in complex phenotypes, it is beneficial to perform module

93 detection using prior knowledge as computational constraints [1, 4, 20]. These methods start

94 from a set of known genes for a given phenotype and expand the module based on the network

95 structure. For example, one of the simplest ways to isolate a functional module by expansion is

96 to assume all the immediate neighbors of the genes associated with the known phenotype are

97 included in the module [1]. However, this method has proven to yield many false positives [1].

98 Therefore, network-based candidate gene prediction algorithms such as the Hishigaki method

99 [21] and label propagation algorithm [22], which have been shown to be more accurate [1, 4, 21], are often used to predict new candidate genes for inclusion in a module.

One purpose of network analysis is the identification of hub genes, which are defined as important genes that are central to the stability of the module [23, 24]. Hub genes have a higher number of interactions (degrees) than other genes in the module. Their removal is most likely to

104 disrupt the module organization, and thus the biological function(s) or phenotype(s) that is 105 governed by the module. Using network analysis, a set of genes for a function or a phenotype 106 can be transformed into a ranked list that is sorted based on their importance in the module.

107 Usually, the number of interactions a gene forms within the module (degree) is used for the 108 ranking [24].

The quality of PPI network data has been a problematic issue in previous network

110 analyses because of the large portion of spurious PPIs generated by experimental methods, such

111 as high-throughput yeast two-hybrid assay [4, 25]. Therefore, in our previous work [25], we 
112 improved the quality of the PPI networks retrieved from the STRING database (STRING,

113 RRID:SCR_005223) [26] by integrating existing experimental knowledge about gene-anatomy

114 relationships available in literature using Uberon anatomy ontology [27]. First, semantic

115 anatomy-based gene networks were generated by calculating the semantic similarity between

116 anatomy terms annotated to different genes, and then, these semantic networks were integrated

117 with the PPI networks for zebrafish and mouse, which improved the candidate gene prediction

118 accuracy for anatomical entities [25]. In this study, we use these improved integrated networks to

119 obtain the most accurate modules.

120 When considering the evolution of functional modules, most studies have focused on

121 identifying the genes that are retained during evolution, i.e., conserved genes, and their

122 organization in the respective modules $[8,10]$. It has been hypothesized that gradual modular

123 changes occur in evolution while maintaining the basic modular structure; this is because

124 dramatic changes in gene interactions may destroy the proper function of an organism [7]. In

125 support of this hypothesis, conserved genes are observed to play an important role in maintaining

126 the stability of the gene modules during evolution $[7,8,10]$. The recruitment and the removal of

127 other genes and the rewiring of biological pathways are often held together by the conserved

128 genes. Performing module analysis allows identification of these important conserved genes,

129 which are often also identified as hub genes [7, 8]. While such conserved module genes may play

130 a role in maintaining gene module structure, species-specific module genes that have been

131 recruited or removed during the evolution may play important roles that contribute to

132 evolutionary transitions [28].

In this work, our goal is to compare PPI network modules associated with fins and limbs

134 to identify the genetic changes, such as the changes in involved genes and their importance, 
135 which led to the anatomical changes that characterize the evolution of fins to limbs. From this

136 analysis, we identify genes that are conserved between fins and limbs to understand their roles in

137 the modular evolution, and we predict novel gene candidates with no previously known

138 contributions to the development of paired fins or paired limbs. Further, we identify fin module-

139 specific and limb module-specific genes and investigate their evolutionary roles. This work

140 suggests some evolutionary hypotheses regarding the role of conserved genes versus fin or limb

141 specific genes in the many evolutionary changes in these animals. Finally, this study

142 demonstrates a general network-based computational model to perform gene module

143 comparisons for evolutionary phenotypic transitions.

\section{2. Methods}

146 (a) Selection of the integrated networks for module detection

147 Based on our previous work [25] of network-based candidate gene prediction using quality-

148 enhanced PPI networks that were generated by four semantic similarity methods (Lin, Resnik,

149 Schlicker, and Wang), the best performing gene networks for zebrafish and the mouse were

150 selected for this project. These are referred to as 'zebrafish integrated network' and 'mouse

151 integrated network' from herein.

153 (b) Detection of network modules

154 For module detection, genes with direct annotations to the pectoral fin (UBERON:0000151),

155 forelimb (UBERON:0002102), pelvic fin (UBERON:0000152), and hindlimb

156 (UBERON:0002103) were used as prior information and their anatomical profiles were extracted

157 from the Monarch Initiative repository (https://monarchinitiative.org/; RRID:SCR_000824) 
$158(06 / 20 / 2018)[29]$. In addition, genes that were annotated to the parts (e.g., pectoral fin

159 lepidotrichium and pectoral fin radial skeleton are parts of the pectoral fin) and the

160 developmental precursors (pectoral fin bud, pelvic fin bud, forelimb bud, and hindlimb bud) of

161 the above entities were extracted using the Uberon anatomy ontology relationships. The genes

162 directly annotated to the anatomical entity of interest or annotated to a part or developmental

163 precursor of the entity are collectively referred to as 'genes with original annotations'.

164 Beginning with the genes with original annotations, gene modules for the anatomical

165 entities of interest were identified by predicting novel genes using the Hishigaki network-based

166 candidate gene prediction method [21, 25]. First, the network-based candidate gene prediction

167 performance for each anatomical entity of interest was evaluated using leave-one-out cross-

168 validation [25], and ROC and precision-recall curves were generated. Then, a prediction

169 precision threshold was used to predict new candidate genes. A trial and error method was used

170 to select the best precision threshold for each gene module.

171 After predicting the candidate genes, the modules were extracted for the pectoral fin and

172 the pelvic fin from the zebrafish integrated network and for the forelimb and the hindlimb from

173 the mouse integrated network. The extracted modules were visualized using the Cytoscape

174 software [30] (Cytoscape, RRID:SCR_003032).

175

176 (c) Validation of the predicted genes

177 The predicted candidate genes could be validated using either experimental methods, such as

178 gene knockdown [31], or computational methods such as the one used in this work. First, the

179 predicted genes for the pectoral fin and pelvic fin modules in zebrafish were compared with the 
orthologous genes in the forelimb and hindlimb modules in mouse and vice versa to determine whether they were annotated to a homologous anatomical entity.

Second, enrichment analyses were performed to confirm for each module, whether the

183 predicted genes shared similar Biological Process terms from Gene Ontology (GO-BP) as the

184 genes with original annotations. Enrichment analyses were also performed to confirm for each

185 module, whether the predicted genes shared similar Uberon anatomy annotations as the genes

186 with original annotations.

Third, the weighted degree distributions of the predicted genes were compared with the weighted degree distributions of the genes with original annotations in each module. If the predicted genes have a higher weighted degree distribution, it indicates that the predicted genes have a similar or a higher importance as genes with original annotations.

(d) Comparison of the network modules

193 To study the fin to limb transition and identify the modular changes, the pectoral fin and pelvic

194 fin modules of the zebrafish were compared with the forelimb and hindlimb modules of the 195 mouse, respectively.

Teleost fishes, such as the zebrafish, have more genes than tetrapods, such as the mouse.

197 A whole genome duplication event is proposed to have occurred at the origin of actinopterygian

198 fishes, i.e., the teleost genome duplication [32]; hence, most of the mouse genes have duplicated

199 copies in the zebrafish. To perform the module comparison, the gene ortholog mappings between

200 mouse and zebrafish genes were retrieved from the Zebrafish Information Network [33] (ZFIN,

201 06/26/2018) (https://zfin.org/downloads) (Zebrafish Information Network, RRID:SCR_002560).

202 During the comparison, if multiple zebrafish orthologs were present in a zebrafish module for a 
single mouse gene, all zebrafish orthologs were retained. By performing the module comparison,

204

205

206

207

208

209

210

211

212

213

214

215

216

217

218

219

220

221

222

223

224

conserved genes (genes that are common to the two modules), zebrafish module-specific genes,

and mouse module-specific genes were identified.

In network analysis, the degree of a gene (the number of interactions of the gene) is often used as an important metric $[10,24]$. Genes with higher degrees in a module, i.e., hub genes, are considered more important because they have more interactions with other module genes and removal of such a gene from the module may significantly affect the integrity of the module [23]. When analyzing networks with weights assigned for interactions (weighted networks), such as the integrated networks used here, weighted degree is preferred over the degree because it considers the different interaction weights rather (equation 1) than counting the number of interactions for a specific node [24].

$$
\text { Weighted degree }=\sum_{v \in n(u)} \operatorname{sim}(v, u)
$$

In equation $1, n(u)$ is the neighborhood of the gene of interest $(u)$ and $v$ iterates through

all the neighbors of gene $u$. The gene similarity score for the interaction between genes $v$ and $u$, which is represented by $\operatorname{sim}(v, u)$, is used for the interaction weight. Weighted degree of gene $u$ is the summation of all weights of interactions between gene $u$ and all its neighbors.

The weighted degree for each gene in a module was calculated, and the genes were ranked accordingly. During the comparisons, the weighted degree of each zebrafish module gene was compared with the corresponding mouse ortholog. However, due to the size differences of the zebrafish and mouse modules, the weighted degree of each gene had to be normalized by the total number of genes in each module. Then, normalized weighted degree distributions for conserved genes, zebrafish module-specific genes, and mouse module-specific genes were 
225 compared for pectoral fin versus forelimb and pelvic fin versus hindlimb to study the relative

226 importance of genes in each group.

The fate of the zebrafish module-specific genes in mouse was investigated by extracting

228 mouse orthologs for the pectoral and pelvic fin module-specific genes and performing

229 enrichment analyses using Uberon and GO-BP terms. Similarly, the roles of the mouse module-

230 specific genes in zebrafish were investigated using zebrafish orthologs for the forelimb and

231 hindlimb module-specific genes. The DAVID (https://david.ncifcrf.gov/) (DAVID,

232 RRID:SCR_001881) online functional enrichment analysis tool was used to perform gene set

233 enrichment analysis using GO-BP terms. DAVID uses Fisher's exact test [34] to perform

234 enrichment analyses. Although the GO is widely used for enrichment analysis, anatomy

235 ontologies are rarely used. To perform enrichment analysis using the Uberon anatomy ontology

236 and Fisher's exact test, a Python program (Uberon enrichment analysis program) was developed

237 and used. Ontology terms with p-values less than 0.05 were considered as enriched terms.

\section{Results and discussion}

240 (a) Selection of the integrated networks for module detection

241 The integrated networks generated using the Lin and Schlicker methods were selected for

242 module detection for zebrafish and mouse, respectively because they outperformed other

243 integrated networks based on the results of our previous work [25]. The zebrafish Lin integrated

244 network contained 17,394 genes and 730,855 interactions and the mouse Schlicker integrated

245 network contained 18,002 genes and 613,671 interactions [25].

247 (b) Detection of network modules 
248 The statistics showing the number of genes with original annotations to each anatomical entity

249 are given in electronic supplementary material, table S1. The total number of genes for the

250 pectoral fin (198) and the forelimb (267) were comparatively similar than the total number of

251 genes for the pelvic fin (15) and the hindlimb (777). Detection of the pelvic fin module was

252 challenging because of the low number of original gene annotations. Unlike the limb

253 development in the mouse, where forelimb and hindlimb buds emerge at the same timepoint, the

254 pelvic fin buds emerge at a much later stage than the pectoral fin bud [35]. This may have been a

255 potential reason for fewer annotations to the pelvic fin; the studied gene disruptions may have

256 killed the larval zebrafish before the pelvic fin develops or the larvae may have been sacrificed at

257 a pre-determined early stage.

258 The ROC and precision-recall curves generated for each anatomical entity during the

259 network-based candidate gene prediction evaluations are given in electronic supplementary

260 material, figures S1 and S2, respectively. According to the curves, all anatomical entities except

261 the pelvic fin show high accuracies for network-based candidate gene predictions (the AUC

262 values of ROC curves were higher than 0.85 ). This shows the high reliability of the network

263 candidate gene predictions. The lower performance for the pelvic fin could be due to its low

264 number of original gene annotations. It has been shown that the prediction accuracy improves

265 with the size of the dataset/number of gene annotations, and anatomical entities with a low

266 number of gene annotations can lead to lower AUC values [36].

267 The statistics for the extracted gene modules are given in electronic supplementary

268 material, table S1. The genes with original annotations that were lost during the module

269 extraction are listed in electronic supplementary material, table S2. A high precision threshold of

$270 \quad 0.7$ was used for candidate gene predictions for pectoral fin, forelimb, and hindlimb modules. 
271 The precision threshold for the pelvic fin was lowered to 0.05 to make the number of genes in

272 the pelvic fin and the forelimb modules approximately similar.

273 The visualizations of the resulting modules for the pectoral fin, pelvic fin, forelimb, and

274 hindlimb are given in electronic supplementary material, figures S3, S4, S5, and S6, respectively.

275 The companion Cytoscape network files for these modules are available in electronic

276 supplementary material, files S1, S2, S3, and S4. The genes in the pectoral fin, pelvic fin,

277 forelimb, and hindlimb modules ranked based on the weighted degree are listed in electronic

278 supplementary material, files S5, S6, S7, and S8, respectively.

279

280

(c) Validation of the predicted genes

281 The list of predicted genes for pectoral fin, pelvic fin, forelimb, and hindlimb modules are given

282 in electronic supplementary material, tables S3, S4, S5, and S6, respectively. Of the 45 predicted

283 genes for the pectoral fin, 14 genes had mouse orthologs that were associated with the forelimb

284 (9 direct annotations, 2 annotations only to the parts or the developmental precursors, and 3

285 predicted genes). Of the 605 predicted genes for the pelvic fin, 78 genes had mouse orthologs

286 that were associated with the hindlimb (46 direct annotations, 20 annotations only to the parts or

287 the developmental precursors, and 12 predicted genes). Of the 18 predicted genes for the

288 forelimb, 6 genes had mouse orthologs that were associated with the pectoral fin ( 2 direct

289 annotations, 1 annotation only to the parts or the developmental precursors, and 3 predicted

290 genes). Of the 32 predicted genes for the hindlimb, 12 genes had mouse orthologs that were

291 associated with the pelvic fin (all 12 were predicted genes). These results indicate that the

292 orthologs of the predicted genes are annotated to homologous anatomical entities, providing a

293 certain level of validation for the predicted genes. 
The enriched GO-BP terms that are common to the predicted genes and genes with

295 original annotations to pectoral fin, pelvic fin, forelimb, and hindlimb are listed in electronic

296 supplementary material, tables S7, S8, S9, and S10, respectively. The enriched Uberon terms that

297 are common to the predicted genes and genes with original annotations to pectoral fin, pelvic fin,

298 forelimb, and hindlimb are listed in electronic supplementary material, tables S11, S12, S13, and

299 S14, respectively. There were several common enriched GO-BP terms for all the modules, some

300 of which were related to paired fins and limbs, such as pectoral fin development, fin

301 development, embryonic limb morphogenesis, embryonic digit morphogenesis. Some of the

302 common enriched Uberon terms, such as median fin fold, ventral fin fold, caudal fin,

303 appendicular skeleton and limb, were related with fin or limb development.

304 The boxplot comparisons of the weighted degree distributions for the predicted genes

305 versus genes with original annotations for the pectoral fin, pelvic fin, forelimb, and hindlimb

306 modules are shown in figure 1. In all the modules, the weighted degree distributions of the

307 predicted genes were higher than the genes with original annotations. This indicates that

308 predicted genes as a group are important in the modules and central to the function of the

309 modules, which supports the biological significance of the predicted genes. 
a

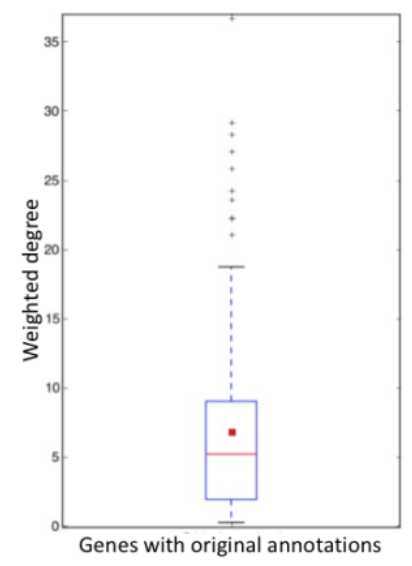

C

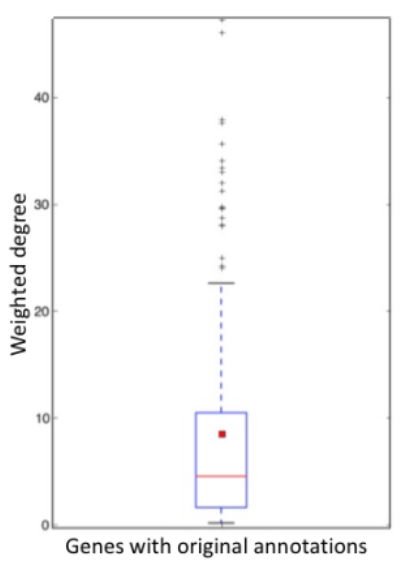

Pectoral fin

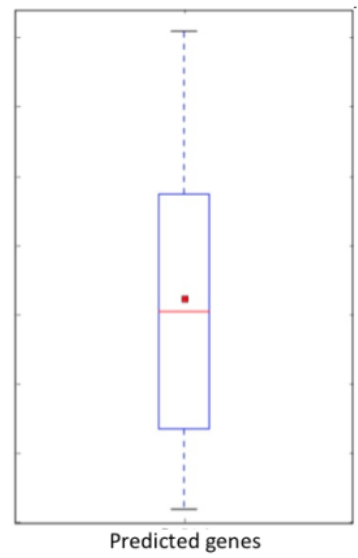

Forelimb

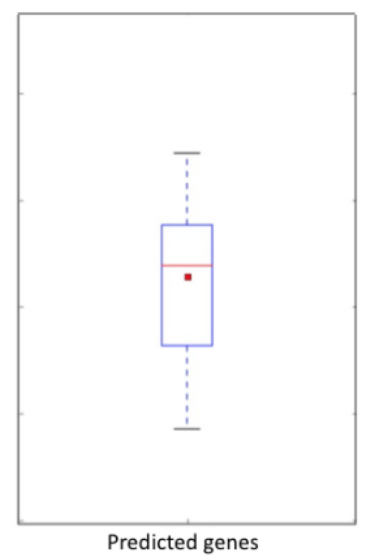

b

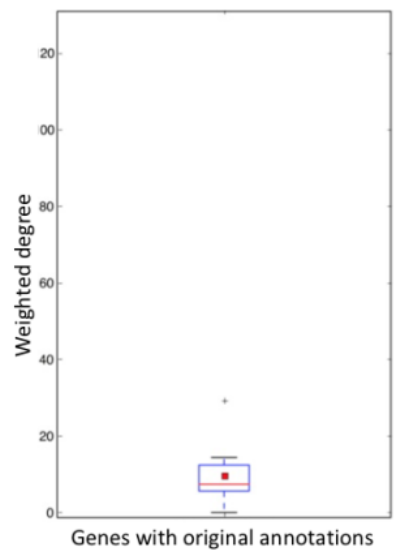

d

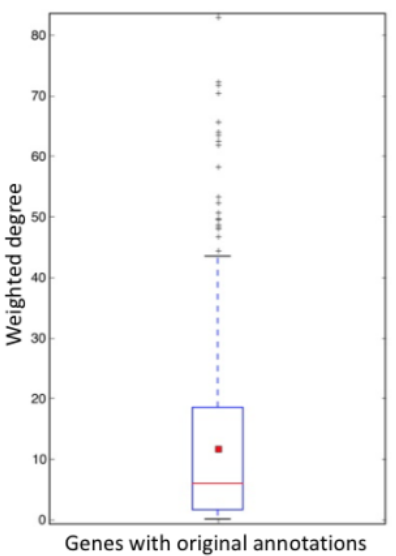

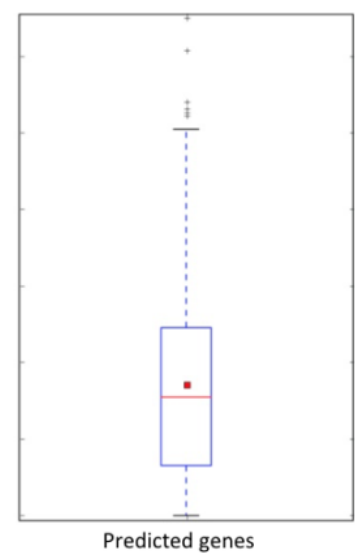

Pelvic fin

Hindlimb

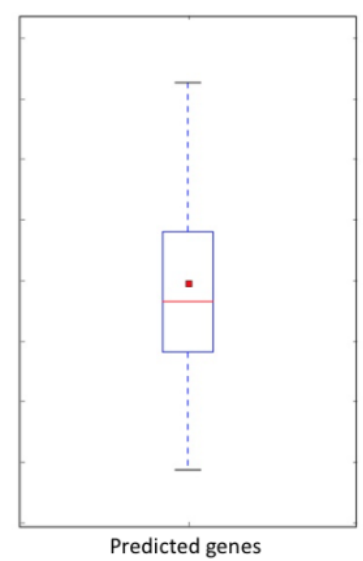

311 Figure 1. The boxplot comparisons of the weighted degree distributions for the predicted genes

312 versus genes with original annotations for each module. In the boxplots, the red line and the

313 square represent the median and mean, respectively.

(d) Comparison of the network modules

316

(i) Pectoral fin and forelimb comparison 
317 According to the comparison, 183 genes were specific to the pectoral fin module, 207 genes

318 were specific to the forelimb module. 37 genes were shared (conserved genes) between the

319 pectoral fin and forelimb (electronic supplementary material, table S15, figure 2).

320 In the pectoral fin module, the top-ranked hub gene based on the weighted degree was

321 shha (sonic hedgehog a) (electronic supplementary material, file S5), whose role has been well-

322 documented in pectoral fin development [16]. Its ortholog, Shh, is important in the development

323 and morphogenesis of limbs in tetrapods including humans [37], and it was also highly ranked in

324 the forelimb module $\left(4^{\text {th }}\right.$, see electronic supplementary material, table S15). The loss or gain of

325 activity in the sonic hedgehog signaling pathway in tetrapods results in lost, gained, or

326 malformed limbs [37]. The shh gene has long been considered an important gene associated with

327 fin to limb transition because it is important in the morphological patterning of paired fins and

$328 \operatorname{limbs}[14]$.

4), another gene closely associated with limb formation and morphogenesis in tetrapods [38].

331 Mutations in bmp4 affect the bmp4 signaling pathway to cause abnormalities in limb and digit

332 formation in tetrapods [38]. Bmp4 was ranked $2^{\text {nd }}$ in the pectoral fin module (electronic

333 supplementary material, table S15) and was predicted during module detection.

When considering the conserved genes (figure 2), some of the important hub genes in the

335 pectoral fin module, such as shha, bmp4, bmp2b, and $b m p 7 a$, had retained their importance

336 demonstrated by their higher ranks based on the weighted degree in the forelimb module

337 (electronic supplementary material, table S15). Other genes such as sox 9 were elevated in rank

338 during the transition from pectoral fin to forelimb. In the pectoral fin module, sox $9 a$ and $\operatorname{sox} 9 b$

339 genes were ranked $83^{\text {rd }}$ and $104^{\text {th }}$, respectively, while in the mouse, the ortholog $\operatorname{sox} 9$ was 
340 elevated to $15^{\text {th }}$ (electronic supplementary material, table S15). Sox9 is well known to be

341 involved with digit patterning in the limbs of tetrapods due to its participation in the a bmp-sox9-

342 wnt Turing network [17, 39]. Because digits emerged after the transition from fins to limbs [13,

$34314]$, the involvement of $\operatorname{sox} 9$ in a digit patterning pathway could have increased the number of

344 interactions with other genes in the forelimb module, and hence, the increased importance.

345 A boxplot comparison of normalized weighted degree distributions for pectoral fin

346 module-specific genes, pectoral fin conserved genes (genes of the pectoral fin in common with

347 forelimb), forelimb conserved genes (genes of the forelimb in common with pectoral fin), and

348 forelimb module-specific genes are given in figure 3 . The conserved genes in both modules have

349 higher normalized weighted degree distributions compared to the respective module-specific

350 genes. This indicates that the conserved genes share more interactions within the module as a

351 group and are more central to modular stability. From an evolutionary point of view, during the

352 transition from pectoral fin to the forelimb, it appears that genes with higher degrees in the

353 pectoral fin module, such as shha, bmp4, were conserved in the forelimb and new forelimb

354 module-specific genes were recruited surrounding those conserved genes. 
paxib

a

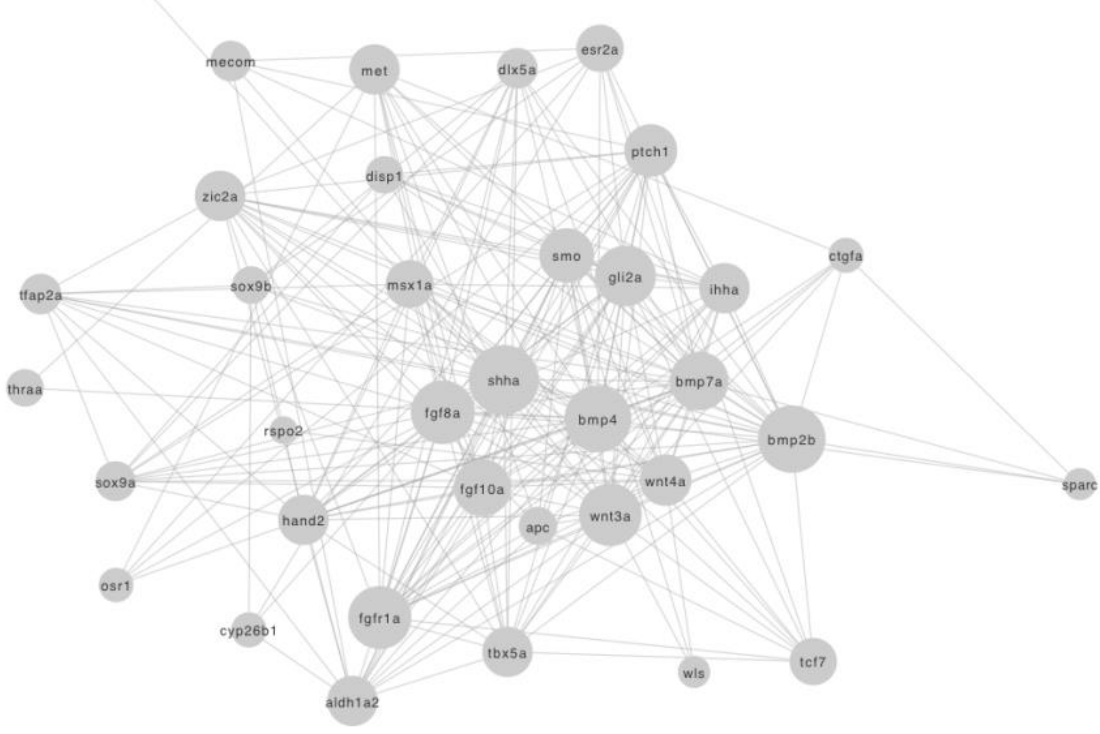

b

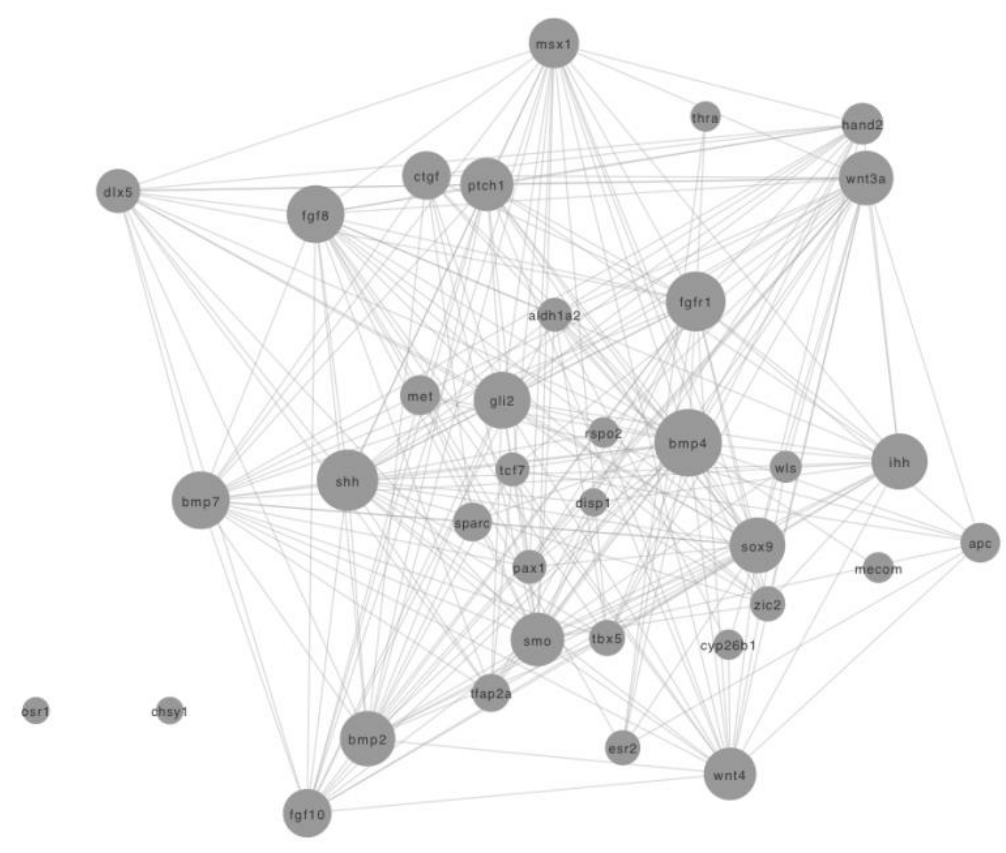

357 Figure 2. Networks of the 37 conserved genes that are common to and extracted from (a) the

358 pectoral fin module and (b) the forelimb module. Node size is proportional to the degree (number 
of interactions) of the gene. Hub genes, such as bmp4, shh, smo, bmp7, sox9, and gli2, are shown in larger node sizes. The arrow represents the direction of modular evolution.

361

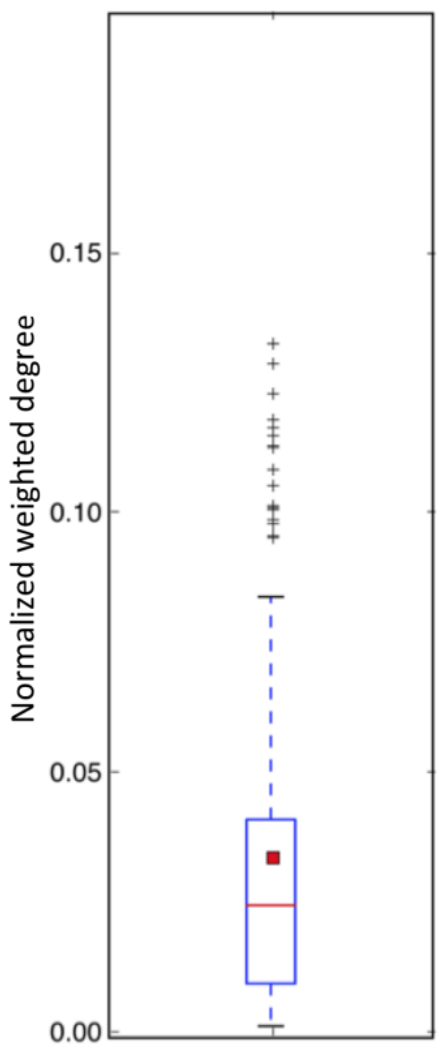

a

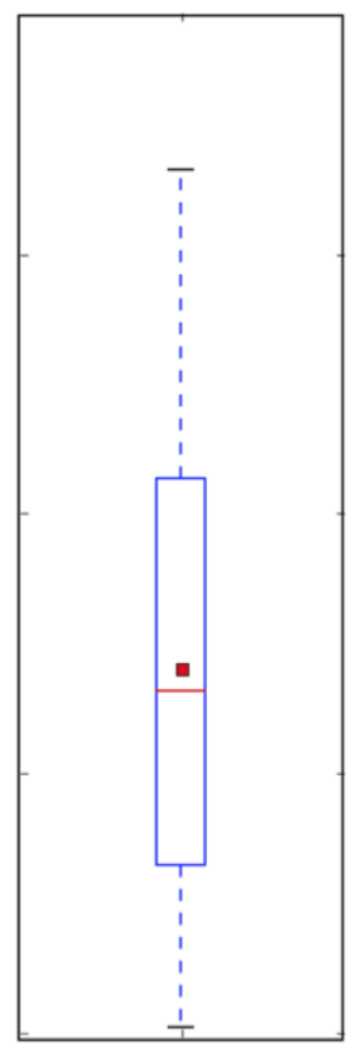

b

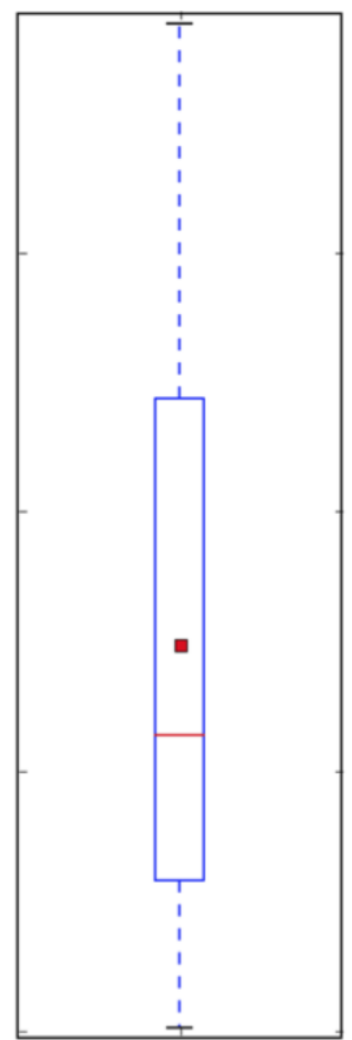

C

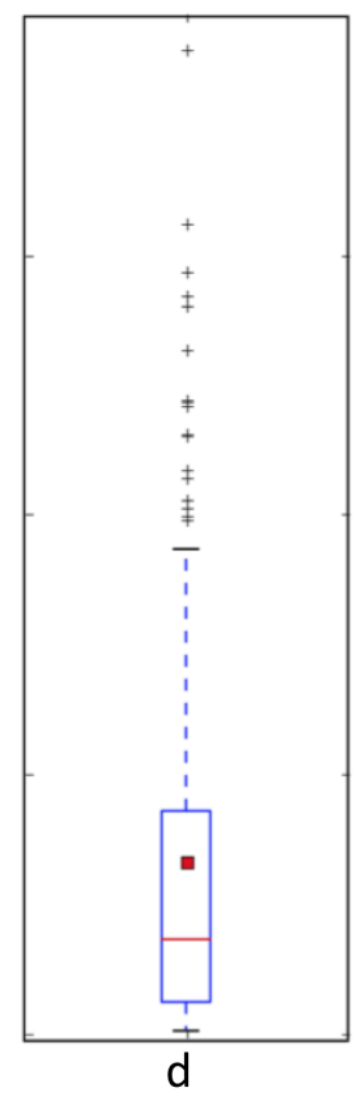

362 Figure 3. Boxplot comparison of normalized weighted degree distributions for (a) pectoral fin

363 module-specific genes, (b) pectoral fin conserved genes, (c) forelimb conserved genes, and (d)

364 forelimb module-specific genes. In the boxplots, the red line and the square represent the median 365 and mean, respectively.

367 (ii) Pelvic fin and hindlimb comparison

368 According to the comparison, 536 genes were specific to the pelvic fin module, 601 genes were specific to the hindlimb module, and 81 genes were conserved between pectoral fin and forelimb modules. (electronic supplementary material, table S16 and figure 4). 
In the pelvic fin module, the highest-ranking gene was $h s p 90 a b$ (predicted) (electronic

372 supplementary material, file S6). Although it is a heat shock protein and does not have known

373 effects on the pelvic fin, studies have shown that the inhibition of its expression causes defects in

374 zebrafish, especially in eye development [40]. Furthermore, the disruption of $h s p 90 a b$ expression

375 has been associated with caudal fin fold defects in the zebrafish [40], which is not recorded in the

376 ZFIN or the Monarch Initiative repository. Our computational results, together with a noted

377 effect on a fin, indicate that $h \mathrm{sp} 90 \mathrm{ab}$ is a prime new candidate gene for pelvic fin development

378 that may have a key role in the module stability.

379 The top ranked hub gene in the hindlimb module based on weighted degree was trp53

380 (electronic supplementary material, file S8), which has been associated with embryonic hindlimb

381 development in mouse [41]. When trp53 is disrupted, mouse limbs are deformed [42]. Trp53 was

382 also found in the pelvic fin module (predicted gene) but it had a lower rank (24th) based on the

383 weighted degree (figure 4 and electronic supplementary material, table S16).

385 (electronic supplementary material, table S16 and figure 4), several that are central to the

386 modular stability were identified. For example, the ctnnbl gene, predicted and ranked $4^{\text {th }}$ in the

387 pelvic fin module, was also highly ranked $\left(3^{\text {rd }}\right)$ in the forelimb module. Ctnnbl is essential for

388 the $\beta$-catenin pathway, which is necessary for the hindlimb initiation in the mouse [43]. Although

389 it does not have known association to either of the paired fins in the zebrafish, it is known to be

390 essential in fish development [44].

391

A boxplot comparison of normalized weighted degree distributions for pelvic fin module-

392 specific genes, pelvic fin conserved genes, hindlimb conserved genes, and hindlimb module-

393 specific genes is given in figure 5. The conserved genes in both modules show higher normalized 
394 weighted degree distributions compared to their respective module-specific genes. As observed

395 for the pectoral fin, this indicates the higher importance of the conserved genes for the stability

396 of the modules.

397

398 (iii) The fate of zebrafish paired fin module-specific genes in the mouse

399 A large number of zebrafish fin module genes (183 for pectoral fin and 536 for pelvic fin) were

400 not included in the mouse limb modules (electronic supplementary material, files S5 and S6),

401 implying these genes had not been maintained in limb development. To understand the roles of

402 those zebrafish pectoral and pelvic fin module-specific genes in the mouse, the enriched GO-BP

403 and Uberon terms for the mouse orthologs for these fin module-specific genes are given in

404 electronic supplementary material, tables S17, S18, S19 and S20. They were enriched for a

405 number of novel anatomical entities and related biological processes unique to tetrapods [13]

406 (electronic supplementary material, tables S21 and S22). 


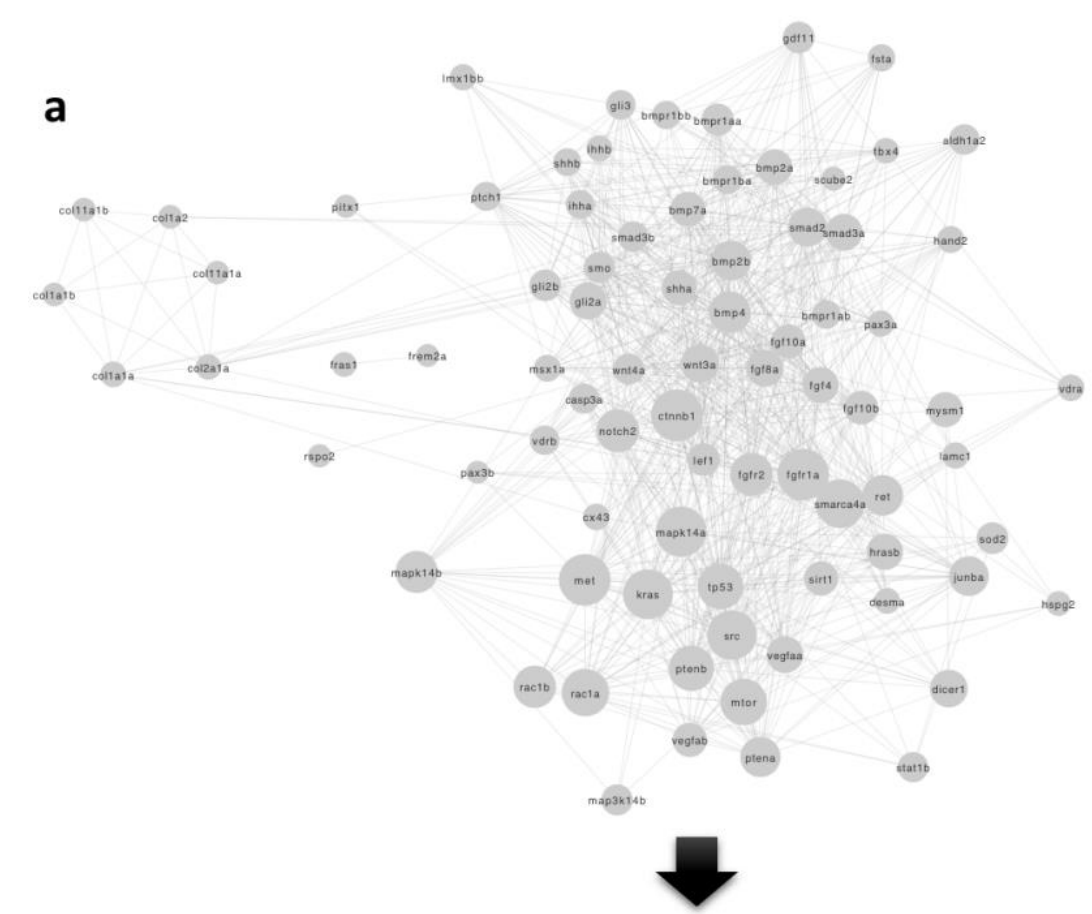

b
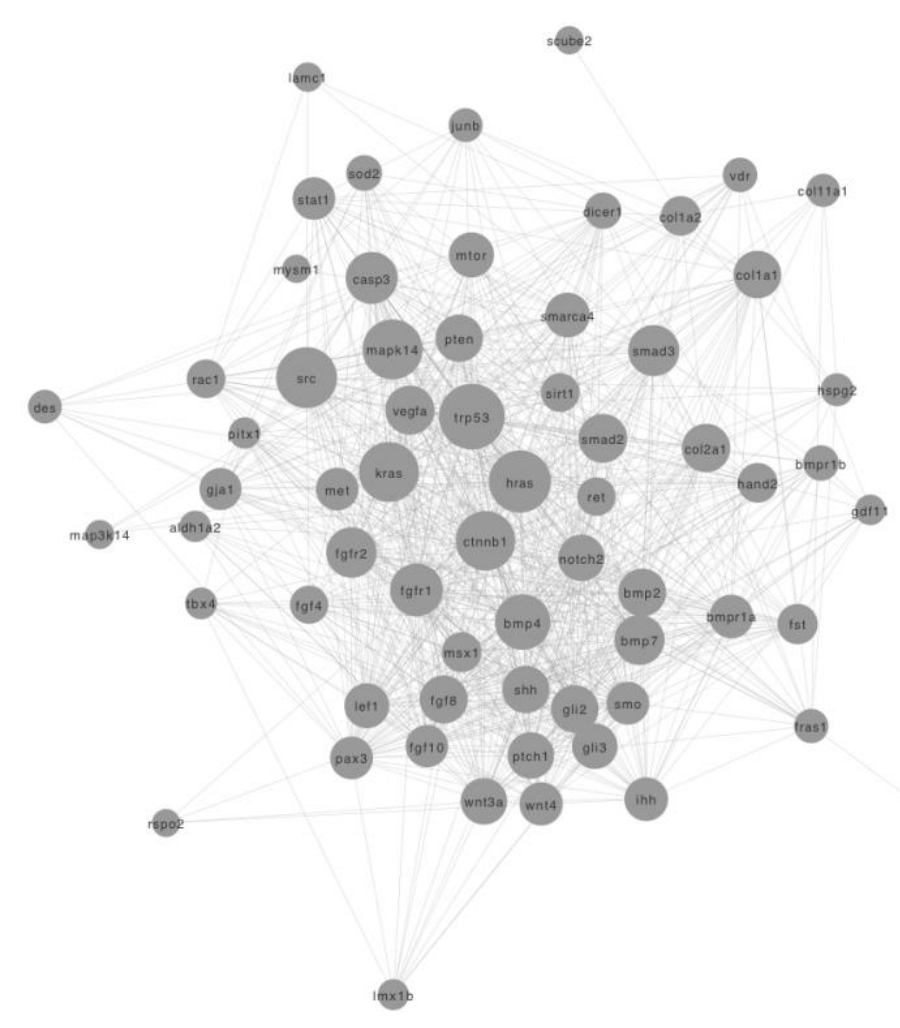

409 Figure 4. Networks of the 81 conserved genes common to and extracted from (a) the pelvic fin module and (b) the hindlimb module. Node size is proportional to the degree (number of 
411 interactions) of the gene. Hub genes, such as $b m p 4, \operatorname{sh}, c t n b 1, b m p 7, \operatorname{trp} 53$, and hras, are shown

412 in larger node sizes. The arrow represents the direction of modular evolution.

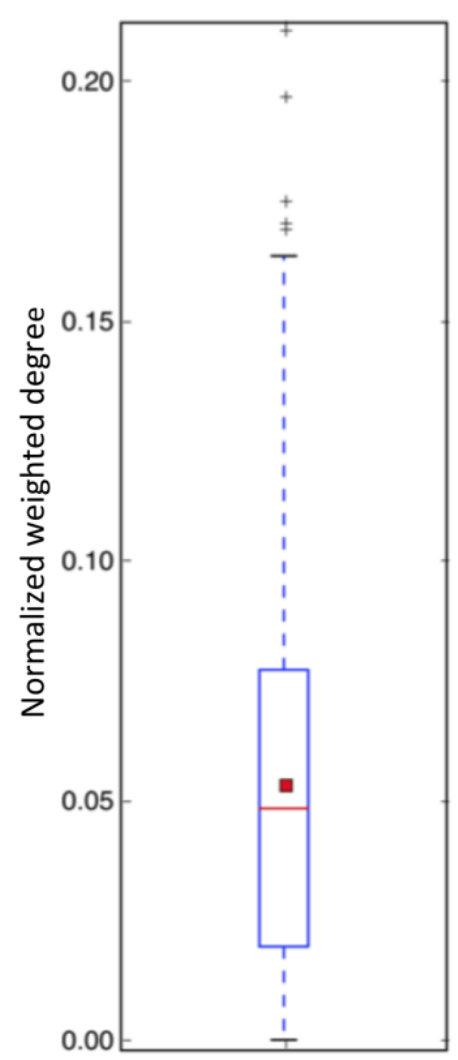

a

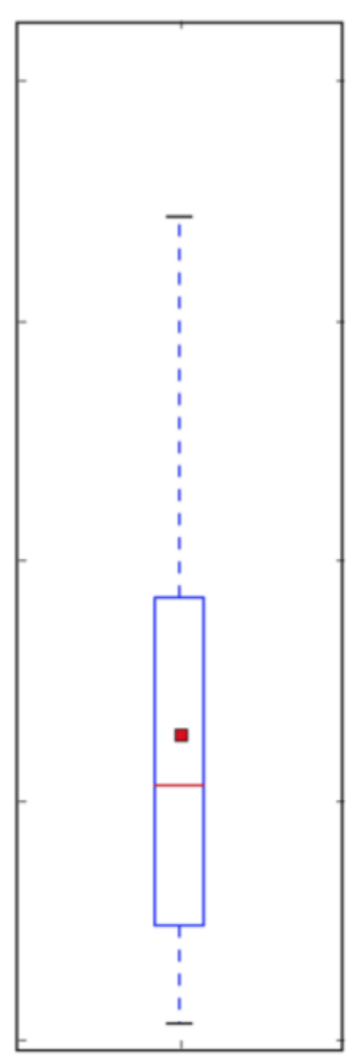

b

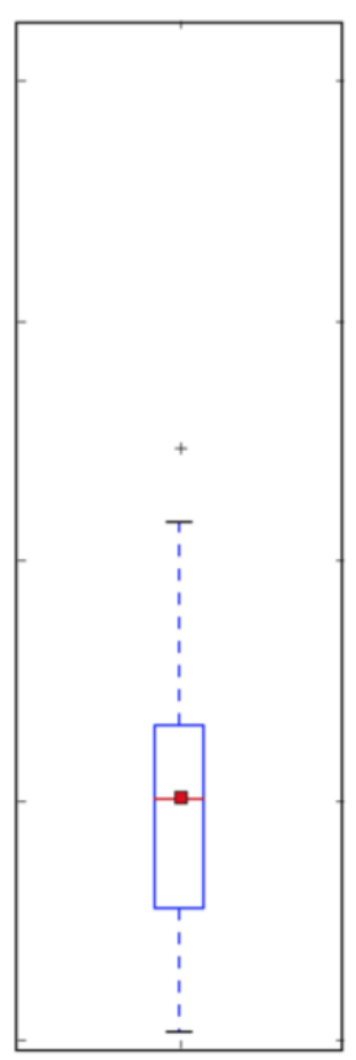

C

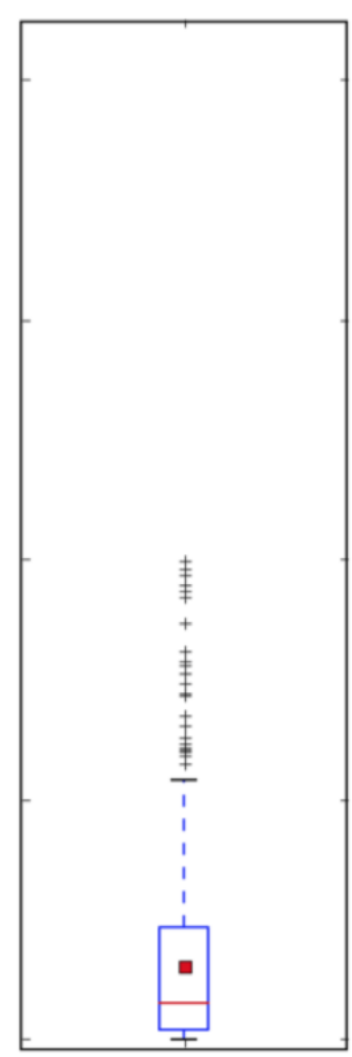

d

414 Figure 5. Boxplot comparison of normalized weighted degree distributions for (a) pelvic fin

415 module-specific genes, (b) pelvic fin conserved genes, (c) hindlimb conserved genes, and (d)

416 hindlimb module-specific genes. In the boxplots, the red line and the square represent the median

417 and mean, respectively.

For instance, the pectoral fin module-specific gene lefl, an important (ranked $7^{\text {th }}$ )

420 member in the pectoral fin module, is involved with palate development, trachea gland

421 development, and associated with neck-related phenotypes [45, 46]. The neck evolved in

422 tetrapods and allowed them to support the head, which was crucial for their success in land [47, 
423 48]. In the pelvic fin module-specific genes, mapkl (ranked 12th) is also associated with neck-

424 related phenotypes, such as thymus development and trachea formation $[49,50]$. It is

425 additionally involved with the lung phenotypes and the development of the lung [50], another

426 structure which progressively evolved in tetrapods that enabled them to breath and thrive in

427 terrestrial environments [51]. Lama5, a gene found in both the pectoral fin and pelvic fin

428 modules, is an example of another module-specific gene that is involved with lung development

429 in the mouse [52]. Furthermore, it is also involved with hair follicle development and hair-related

430 phenotypes [53], which are other anatomical entities specific for mammals [54]. These examples

431 point to the possibility that many of genes used in fin development were recruited in the

432 development of novel anatomical entities that enabled tetrapods to thrive in a terrestrial

433 environment.

435 (iv) The role of mouse module-specific limb genes in the zebrafish

436 A large number of module-specific genes for the forelimb and hindlimb (207 for forelimb and

437601 for hindlimb) did not appear in pectoral fin or pelvic fin modules (electronic supplementary

438 material, files S7 and S8), and the question of their developmental function in the zebrafish

439 occurred. To understand the function of the limb module-specific genes in zebrafish, the

440 enriched GO-BP and Uberon terms for the limb module-specific genes are given in electronic

441 supplementary material, tables S23, S24, S25 and S26. According to the enrichment analyses,

442 these mouse limb module-specific genes were enriched to the head of the zebrafish, specifically,

443 the jaw skeleton and post-hyoid pharyngeal arch skeleton (electronic supplementary material,

444 tables S27 and S28). The latter region includes the gill chamber and contains parts such as gill

445 rakers [55] that have been lost in tetrapods. For instance, $f_{s t}$ is a crucial forelimb module-specific 
446 gene, which has a zebrafish ortholog $(f s t a)$ with phenotypes related to splanchnocranium [56]

447 and post-hyoid pharyngeal arch skeleton [57] that supports the gill chamber. Furthermore, twist1

448 is module-specific for both forelimb and hindlimb, and it has two zebrafish orthologs (twist la

449 and twist $1 b$ ) that are involved with pharyngeal system development [58].

450 There are some mouse module-specific genes, e.g., $\operatorname{tg} f b r 3$, which are involved in the

451 development of both the forelimb and the hindlimb, that is associated with the development of

452 the caudal fin in zebrafish [59]. Another example, lep, which is module-specific for both

453 forelimb and hindlimb, is associated with otolith development in zebrafish [60]. Otoliths are

454 located in the inner ear cavity of all teleost fishes where they aid in hearing and serve as balance

455 organs [61]. The enrichment analyses point to the possibility that genes associated with various

456 fish-specific structures such as gill arches and the caudal fin, were recruited for limb

457 development as they were lost during the transition to tetrapods.

4. Conclusion

460 The goal of this work was to study the modular changes associated with the fin to limb transition

461 using gene networks. This computational study expanded the number of genes that could be

462 analyzed compared to wet lab methods and enabled the study of gene network structure rather

463 than individual genes. Employing the quality-enhanced integrated networks ensured that the

464 module detections, gene predictions, and identification of important genes in the modules were

465 accurate, as evidenced from the results. To our knowledge, this is the first work that uses PPI

466 networks to study the fin to limb transition. We discovered important information such as the

467 hub genes responsible for the stability of paired fin and limb modules and changes in the

468 importance of module genes associated with the transition. Some of the module genes were 
469 predicted during the module detection, with evidence confirming their involvement with the

470 respective fins or limbs. This paper tabulates rankings of module genes based on their

471 importance, predicted candidates, and comparisons of the importance of the module genes,

472 which will be useful for future studies on fin to limb transition.

473 Furthermore, we discovered that the conserved genes were more likely to be hub genes

474 than the module-specific genes. Thus, it appeared that during the fin to limb transition, most of

475 the crucial hub genes of fin modules were conserved in the limb, and limb-specific genes were

476 recruited to surround this conserved 'appendage' core network. Moreover, our data suggested

477 that zebrafish fin module-specific genes were additionally employed in anatomical structures that

478 emerged after the aquatic to terrestrial vertebrate transition, such as lung and neck. Furthermore,

479 the evidence implied that mouse limb module-specific genes were involved with anatomical

480 structures, such as the gill rakers in the zebrafish that were lost during the transition. These

481 results provide the groundwork for evolutionary developmental biologists to experimentally

482 investigate aforementioned hypotheses. Most importantly, this work demonstrates how gene

483 networks can be used to study evolutionary phenotypic transitions and this computational

484 workflow can be used to perform large-scale network analyses to study evolutionary transitions

485 involving any model organism and anatomical entity with sufficient data, which is a valuable

486 addition to evolutionary biology.

\section{Data accessibility}

489 The network files and the anatomy profiles used for the candidate gene predictions are available 490 at https://doi.org/10.6084/m9.figshare.13589579.v1 and the Python scripts used for this analysis 491 are available at https://doi.org/10.5281/zenodo.4445583. 


\section{Authors' contributions}

494 All authors planned and designed the experiments. PCF wrote the Python scripts for the analysis 495 and performed the experiments under the supervision of PMM and EZ. All authors analyzed the 496 results, read and approved the final manuscript.

\section{Competing interests}

499 The authors declare that they have no competing interests. The founding sponsors played no role 500 in the design of this study; the collection, analyses or interpretation of the data; the writing of the 501 manuscript; or the decision to publish the results.

\section{$503 \quad$ Funding}

504 This work was supported by the National Science Foundation (NSF) collaborative grant DBI505 1062542, the Phenotype Research Coordination Network (NSF 0956049) and partially by NSF

506 EPSCoR grant IIA-1355423. This work was also supported by the University of Iowa faculty 507 startup fund to EZ. The High-Performance Computing clusters used in this work were supported

508 by an NSF grant OAC-1626516. The views expressed in this paper do not necessarily reflect

509 those of the NSF. Furthermore, PCF received support from the University of South Dakota

510 (USD) Graduate Academic and Creative Research Grant and the Nelson Fellowship from

\section{Biology Department.}


The authors thank J. P. Balhoff for assisting with the retrieval of gene-anatomical entity

515

relationships from the Monarch Initiative repository. The authors thank T. J. Vision, D.

Goodman, B. Wone, W. M. Dahdul, and L. M. Jackson for their assistance and helpful

comments to improve this research.

\section{References}

[1] Cowen, L., Ideker, T., Raphael, B.J. \& Sharan, R. 2017 Network propagation: a universal amplifier of genetic associations. Nature Reviews Genetics.

[2] Yamada, T. \& Bork, P. 2009 Evolution of biomolecular networks [mdash] lessons from metabolic and protein interactions. Nat Rev Mol Cell Biol 10, 791-803.

(doi:http://www.nature.com/nrm/journal/v10/n11/suppinfo/nrm2787_S1.html). [3] Defoort, J., Van de Peer, Y. \& Vermeirssen, V. 2018 Function, dynamics and evolution of network motif modules in integrated gene regulatory networks of worm and plant. Nucleic Acids Res.

[4] Sharan, R., Ulitsky, I. \& Shamir, R. 2007 Network-based prediction of protein function. Molecular systems biology 3, 88 .

[5] Tripathi, S., Moutari, S., Dehmer, M. \& Emmert-Streib, F. 2016 Comparison of module detection algorithms in protein networks and investigation of the biological meaning of predicted modules. BMC bioinformatics 17, 129. (doi:10.1186/s12859-016-0979-8).

[6] Tang, X., Wang, J., Liu, B., Li, M., Chen, G. \& Pan, Y. 2011 A comparison of the functional modules identified from time course and static PPI network data. BMC bioinformatics 12, 339. [7] Vespignani, A. 2003 Evolution thinks modular. Nat. Genet. 35. (doi:10.1038/ng1003-118). [8] Wuchty, S., Oltvai, Z.N. \& Barabási, A.L. 2003 Evolutionary conservation of motif constituents in the yeast protein interaction network. Nat. Genet. 35. (doi:10.1038/ng1242). [9] Alhindi, T., Zhang, Z., Ruelens, P., Coenen, H., Degroote, H., Iraci, N. \& Geuten, K. 2017 Protein interaction evolution from promiscuity to specificity with reduced flexibility in an increasingly complex network. Scientific reports 7, 44948. [10] Shui, Y. \& Cho, Y.-R. 2016 Alignment of PPI Networks Using Semantic Similarity for Conserved Protein Complex Prediction. IEEE transactions on nanobioscience 15, 380-389. [11] García del Valle, E.P., Lagunes García, G., Prieto Santamaría, L., Zanin, M., Menasalvas Ruiz, E. \& Rodríguez-González, A. 2019 Disease networks and their contribution to disease understanding: A review of their evolution, techniques and data sources. J. Biomed. Inf. 94, 103206. (doi:https://doi.org/10.1016/j.jbi.2019.103206).

[12] Cheng, F., Jia, P., Wang, Q., Lin, C.-C., Li, W.-H. \& Zhao, Z. 2014 Studying Tumorigenesis through Network Evolution and Somatic Mutational Perturbations in the Cancer Interactome. Molecular Biology and Evolution 31, 2156-2169. (doi:10.1093/molbev/msu167). [13] Shubin, N. 2008 Your inner fish: a journey into the 3.5-billion-year history of the human body, Vintage.

[14] Amaral, D.B. \& Schneider, I. 2018 Fins into limbs: Recent insights from sarcopterygian fish. genesis 56, e23052. 
[15] Vorobyeva, E.I. \& Schultze, H.-P. 1991 Description and systematics of panderichthyid fishes with comments on their relationship to tetrapods. Origins of the higher groups of tetrapods: controversy and consensus, 68-109. [16] Letelier, J., de la Calle-Mustienes, E., Pieretti, J., Naranjo, S., Maeso, I., Nakamura, T., Pascual-Anaya, J., Shubin, N.H., Schneider, I., Martinez-Morales, J.R., et al. 2018 A conserved Shh cis-regulatory module highlights a common developmental origin of unpaired and paired fins. Nat. Genet. 50, 504-509. (doi:10.1038/s41588-018-0080-5).

[17] Onimaru, K., Marcon, L., Musy, M., Tanaka, M. \& Sharpe, J. 2016 The fin-to-limb transition as the re-organization of a Turing pattern. Nature communications 7, 11582. [18] Zeng, E., Ding, C., Mathee, K., Schneper, L. \& Narasimhan, G. 2012 Gene Function Prediction and Functional Network: The Role of Gene Ontology. In Data Mining: Foundations and Intelligent Paradigms (eds. D.E. Holmes \& L.C. Jain), pp. 123-162, Springer Berlin Heidelberg.

[19] Kernighan, B.W. \& Lin, S. 1970 An efficient heuristic procedure for partitioning graphs. The Bell system technical journal 49, 291-307.

[20] Zeng, E., Yang, C., Li, T. \& Narasimhan, G. Year On the effectiveness of constraints sets in clustering genes. IEEE.

[21] Hishigaki, H., Nakai, K., Ono, T., Tanigami, A. \& Takagi, T. 2001 Assessment of prediction accuracy of protein function from protein-protein interaction data. Yeast 18, 523-531. (doi:10.1002/yea.706).

[22] Liu, X. \& Murata, T. 2010 Advanced modularity-specialized label propagation algorithm for detecting communities in networks. Physica A: Statistical Mechanics and its Applications 389, 1493-1500.

[23] Taylor, I.W., Linding, R., Warde-Farley, D., Liu, Y., Pesquita, C., Faria, D., Bull, S., Pawson, T., Morris, Q. \& Wrana, J.L. 2009 Dynamic modularity in protein interaction networks predicts breast cancer outcome. Nat Biotech 27, 199-204.

(doi:http://www.nature.com/nbt/journal/v27/n2/suppinfo/nbt.1522_S1.html).

[24] Tang, X., Wang, J., Zhong, J. \& Pan, Y. 2014 Predicting essential proteins based on weighted degree centrality. IEEE/ACM Transactions on Computational Biology and Bioinformatics (TCBB) 11, 407-418.

[25] Fernando, P.C., Mabee, P.M. \& Zeng, E. 2020 Integration of anatomy ontology data with protein-protein interaction networks improves the candidate gene prediction accuracy for anatomical entities. BMC bioinformatics 21, 442. (doi:10.1186/s12859-020-03773-2). [26] Szklarczyk, D., Morris, J.H., Cook, H., Kuhn, M., Wyder, S., Simonovic, M., Santos, A., Doncheva, N.T., Roth, A., Bork, P., et al. 2017 The STRING database in 2017: qualitycontrolled protein-protein association networks, made broadly accessible. Nucleic Acids Res. $\mathbf{4 5}$, D362-D368. (doi:10.1093/nar/gkw937).

[27] Mungall, C.J., Torniai, C., Gkoutos, G.V., Lewis, S.E. \& Haendel, M.A. 2012 Uberon, an integrative multi-species anatomy ontology. Genome biology 13, R5. (doi:10.1186/gb-2012-13$1-\mathrm{r} 5)$.

[28] Liang, Z., Xu, M., Teng, M. \& Niu, L. 2006 Comparison of protein interaction networks reveals species conservation and divergence. BMC bioinformatics 7, 457.

[29] Mungall, C.J., McMurry, J.A., Köhler, S., Balhoff, J.P., Borromeo, C., Brush, M., Carbon, S., Conlin, T., Dunn, N., Engelstad, M., et al. 2017 The Monarch Initiative: an integrative data and analytic platform connecting phenotypes to genotypes across species. Nucleic Acids Res. 45, D712-D722. (doi:10.1093/nar/gkw1128). 
601

602

603

604

605

606

607

608

609

610

611

612

613

614

615

616

617

618

619

620

621

622

623

624

625

626

627

628

629

630

631

632

633

634

635

636

637

638

639

640

641

642

643

644

645

[30] Smoot, M.E., Ono, K., Ruscheinski, J., Wang, P.-L. \& Ideker, T. 2011 Cytoscape 2.8: new features for data integration and network visualization. Bioinformatics 27, 431-432.

[31] Huang, L., Jin, J., Deighan, P., Kiner, E., McReynolds, L. \& Lieberman, J. 2013 Efficient and specific gene knockdown by small interfering RNAs produced in bacteria. Nature biotechnology 31, 350-356.

[32] Meyer, A. \& Schartl, M. 1999 Gene and genome duplications in vertebrates: the one-to-four (-to-eight in fish) rule and the evolution of novel gene functions. Current opinion in cell biology 11, 699-704.

[33] Bradford, Y., Conlin, T., Dunn, N., Fashena, D., Frazer, K., Howe, D.G., Knight, J., Mani, P., Martin, R., Moxon, S.A.T., et al. 2011 ZFIN: enhancements and updates to the zebrafish model organism database. Nucleic Acids Res. 39, D822-D829. (doi:10.1093/nar/gkq1077).

[34] Routledge, R. 2005 Fisher's exact test. Encyclopedia of biostatistics.

[35] Grandel, H. \& Schulte-Merker, S. 1998 The development of the paired fins in the Zebrafish (Danio rerio). Mechanisms of Development 79, 99-120. (doi:https://doi.org/10.1016/S09254773(98)00176-2).

[36] Varoquaux, G. 2018 Cross-validation failure: small sample sizes lead to large error bars. Neuroimage 180, 68-77.

[37] Lopez-Rios, J. 2016 The many lives of SHH in limb development and evolution. Seminars in Cell \& Developmental Biology 49, 116-124.

(doi:https://doi.org/10.1016/j.semcdb.2015.12.018).

[38] Bakrania, P., Efthymiou, M., Klein, J.C., Salt, A., Bunyan, D.J., Wyatt, A., Ponting, C.P., Martin, A., Williams, S., Lindley, V., et al. 2008 Mutations in BMP4 Cause Eye, Brain, and Digit Developmental Anomalies: Overlap between the BMP4 and Hedgehog Signaling Pathways. The American Journal of Human Genetics 82, 304-319.

(doi:https://doi.org/10.1016/j.ajhg.2007.09.023).

[39] Raspopovic, J., Marcon, L., Russo, L. \& Sharpe, J. 2014 Digit patterning is controlled by a Bmp-Sox9-Wnt Turing network modulated by morphogen gradients. Science 345, 566-570. [40] Yeyati, P.L., Bancewicz, R.M., Maule, J. \& van Heyningen, V. 2007 Hsp90 Selectively Modulates Phenotype in Vertebrate Development. PLOS Genetics 3, e43. (doi:10.1371/journal.pgen.0030043).

[41] Im, C.H., Chau, K., Lopez, M., Sun, X.Z., Tran, J., Cuya, S. \& Sharp, S.B. 2016 Hindlimb Muscles of 17.5-18.5 dpc Mice Double Null for MyoD and Trp53 Appear Indistinguishable from Muscles of Mice Null for Either Gene. The FASEB Journal 30, 1035-1032.

[42] Vares, G., Wang, B., Tanaka, K., Shang, Y., Fujita, K., Hayata, I. \& Nenoi, M. 2011 Trp53 activity is repressed in radio-adapted cultured murine limb bud cells. Journal of radiation research 52, 727-734.

[43] Kawakami, Y., Marti, M., Kawakami, H., Itou, J., Quach, T., Johnson, A., Sahara, S., O’Leary, D.D.M., Nakagawa, Y. \& Lewandoski, M. 2011 Islet1-mediated activation of the $\beta$ catenin pathway is necessary for hindlimb initiation in mice. Development 138, 4465-4473. [44] Zhang, M., Zhang, J., Lin, S.-C. \& Meng, A. $2012 \beta$-Catenin 1 and $\beta$-catenin 2 play similar and distinct roles in left-right asymmetric development of zebrafish embryos. Development, dev074435 .

[45] Duan, D., Yue, Y., Zhou, W., Labed, B., Ritchie, T.C., Grosschedl, R. \& Engelhardt, J.F. 1999 Submucosal gland development in the airway is controlled by lymphoid enhancer binding factor 1 (LEF1). Development 126, 4441-4453. 
646

[46] Nawshad, A. \& Hay, E.D. 2003 TGFß3 signaling activates transcription of the LEF1 gene to induce epithelial mesenchymal transformation during mouse palate development. The Journal of cell biology 163, 1291-1301.

[47] Sallan, L.C. 2012 Tetrapod-like axial regionalization in an early ray-finned fish.

Proceedings of the Royal Society B: Biological Sciences 279, 3264-3271.

(doi:10.1098/rspb.2012.0784).

[48] Ericsson, R., Knight, R. \& Johanson, Z. 2013 Evolution and development of the vertebrate neck. J. Anat. 222, 67-78. (doi:10.1111/j.1469-7580.2012.01530.x).

[49] Hoffman, B.G., Williams, K.L., Tien, A.H., Lu, V., de Algara, T.R., Ting, J.-y. \& Helgason, C.D. 2006 Identification of novel genes and transcription factors involved in spleen, thymus and immunological development and function. Genes and immunity 7, 101.

[50] Boucherat, O., Nadeau, V., Bérubé-Simard, F.-A., Charron, J. \& Jeannotte, L. 2014 Crucial requirement of ERK/MAPK signaling in respiratory tract development. Development 141, 3197. [51] Tatsumi, N., Kobayashi, R., Yano, T., Noda, M., Fujimura, K., Okada, N. \& Okabe, M. 2016 Molecular developmental mechanism in polypterid fish provides insight into the origin of vertebrate lungs. Scientific Reports 6, 30580. (doi:10.1038/srep30580). [52] Nguyen, N.M., Miner, J.H., Pierce, R.A. \& Senior, R.M. 2002 Laminin $\alpha 5$ is required for lobar septation and visceral pleural basement membrane formation in the developing mouse lung. Developmental biology 246, 231-244.

[53] Gao, J., DeRouen, M.C., Chen, C.-H., Nguyen, M., Nguyen, N.T., Ido, H., Harada, K., Sekiguchi, K., Morgan, B.A. \& Miner, J.H. 2008 Laminin-511 is an epithelial message promoting dermal papilla development and function during early hair morphogenesis. Genes $\&$ development 22, 2111-2124.

[54] Wu, D.-D., Irwin, D.M. \& Zhang, Y.-P. 2008 Molecular evolution of the keratin associated protein gene family in mammals, role in the evolution of mammalian hair. BMC Evolutionary

Biology 8, 241. (doi:10.1186/1471-2148-8-241).

[55] Gillis, J.A., Modrell, M.S. \& Baker, C.V.H. 2013 Developmental evidence for serial homology of the vertebrate jaw and gill arch skeleton. Nature communications 4, 1436.

[56] Dalcq, J., Pasque, V., Ghaye, A., Larbuisson, A., Motte, P., Martial, J.A. \& Muller, M. 2012 RUNX3, EGR1 and SOX9B Form a Regulatory Cascade Required to Modulate BMP-Signaling during Cranial Cartilage Development in Zebrafish. PLOS ONE 7, e50140.

(doi:10.1371/journal.pone.0050140).

[57] Dal-Pra, S., Fürthauer, M., Van-Celst, J., Thisse, B. \& Thisse, C. 2006 Noggin1 and Follistatin-like2 function redundantly to Chordin to antagonize BMP activity. Developmental Biology 298, 514-526. (doi:https://doi.org/10.1016/j.ydbio.2006.07.002).

[58] Das, A. \& Crump, J.G. 2012 Bmps and Id2a Act Upstream of Twist1 To Restrict Ectomesenchyme Potential of the Cranial Neural Crest. PLOS Genetics 8, e1002710. (doi:10.1371/journal.pgen.1002710).

[59] Kamaid, A., Molina-Villa, T., Mendoza, V., Pujades, C., Maldonado, E., Ispizua Belmonte, J.C. \& López-Casillas, F. 2015 Betaglycan knock-down causes embryonic angiogenesis defects in zebrafish. genesis 53, 583-603. (doi:10.1002/dvg.22876).

[60] Liu, Q., Dalman, M., Chen, Y., Akhter, M., Brahmandam, S., Patel, Y., Lowe, J., Thakkar, M., Gregory, A.-V., Phelps, D., et al. 2012 Knockdown of leptin A expression dramatically alters zebrafish development. General and Comparative Endocrinology 178, 562-572. (doi:https://doi.org/10.1016/j.ygcen.2012.07.011). 
bioRxiv preprint doi: https://doi.org/10.1101/2021.01.28.428646; this version posted January 29, 2021. The copyright holder for this preprint (which was not certified by peer review) is the author/funder, who has granted bioRxiv a license to display the preprint in perpetuity. It is made available under aCC-BY 4.0 International license.

691 [61] Rodríguez Mendoza, R.P. 2006 Otoliths and their applications in fishery science. Croatian 692 Journal of Fisheries: Ribarstvo 64, 89-102.

693 